\title{
The Patient Care Pathway for Type 2 Diabetes Mellitus in Asian Countries: Implications on Target Physician Group for Diabetes Clinical Trials
}

\author{
Lansang $\mathrm{EZ}^{1^{\star}}$, Tang $\mathrm{V}^{1}$, Ranade $\mathrm{S}^{2}$, Caveney $\mathrm{E}^{3}$, Ng NK${ }^{1}$, Saldanha $\mathrm{LM}^{1}$, Lee $\mathrm{KJ}^{4}$ and Wai $\mathrm{K}^{1}$
}

${ }^{1}$ Site Services, Quintiles East Asia Pte Ltd, Singapore

${ }^{2}$ Medical and Scientific Services, Quintiles East Asia Pte Ltd, Singapore

${ }^{3}$ Diabetes, Cardiovascular and Metabolic Delivery Unit, Quintiles, USA

${ }^{4}$ Clinical Operations, Quintiles East Asia Pte Ltd, Singapore

\begin{abstract}
Introduction: A patient care pathway is the referral pathway from a patient's first consult with a healthcare provider to the time they receive the most appropriate treatment for their condition. With the increase in diabetes prevalence, clinical trial growth in Asia, and lack of published literature in this area, it is critical to understand the patient care pathway of Type 2 Diabetes Mellitus in the region.

Method: Between March and August 2013, a survey investigating the diagnosis and treatment patterns of diabetes and diabetes-related complications was sent to healthcare offices in 11 Asian countries. Survey responses received were analyzed.

Results: Eighty-seven healthcare offices were contacted, out of which, 49, returned the surveys. Fifty-seven percent of physicians who responded were endocrinologists, $24 \%$ were self- reported diabetologists, and $14 \%$ were general practitioners. Internists/general practitioners diagnose majority of type 2 diabetics, and they manage majority of diabetes patients treated only with lifestyle therapy. Internists and endocrinologists manage most patients on oral anti-hyperglycemic agents. Endocrinologists/diabetologists treat a majority of patients on insulin and GLP-1 agonists. Specialists such as cardiologists and nephrologists manage those with more severe complications.

Discussion: The diabetes patient care pathway has several suggestions on the target physician group for clinical trials, namely (1) Internal medicine doctors and primary healthcare physicians may be potential investigators or referral sites for clinical trials requiring treatment naïve diabetic patients; (2) diabetologists/endocrinologists should be considered for clinical trials needing patients on pharmacologic treatment, whether oral or insulin; (3) internists may play a role in trials that involve patients on oral anti-hyperglycemic agents and on insulin; (4) the severity of diabetic complications impacts the medical specialty of the physician treating patients; and (5) cardiologists and nephrologists are important considerations for studies with cardiovascular or nephrology related outcomes.
\end{abstract}

Keywords: Feasibility; Site identification; Asia; Speciality

\section{Introduction}

Knowledge and awareness of patient's journeys from their first interaction with health care systems to the time they receive appropriate treatment and follow-up help ensure patients receive the correct, efficient treatment [1,2]. In this research, a patient care pathway is defined as the referral pathway from a patient's first consult with a healthcare provider to the time that they receive the most appropriate treatment. Understanding a patient care pathway is crucial for both clinical and commercialization reasons. From a clinical research perspective, a well-defined patient pathway is an effective tool in identifying the right type or types of principal investigators. Furthermore, a culturally-correct, accurate patient pathway allows precise targeting of appropriate departments/ physicians for individual products [1] ensuring the correct patient population receives new therapies. This is specifically beneficial in the management of complex, chronic diseases such as both Type 1 and 2 diabetes mellitus, especially given the plethora of new treatments recently approved or in various development stages [3].

Type 2 diabetes mellitus is increasing globally [4] and specifically in Asia [5]. In Asia, Type 2 diabetes continues to increase at a rapid rate over short periods and develops at a younger age $[5,6]$. Furthermore, with the continued increase in the prevalence of diabetes, Asian countries have over 200 million people with diabetes [7], contributing to more than $60 \%$ of the world's diabetic population $[6,8]$. The estimated prevalence of diabetes among a representative sample of Chinese adults was $11.6 \%$ and the prevalence of prediabetes was $50.1 \%$.
Projections based on sample weighting suggest this may represent up to 113.9 million Chinese adults with diabetes and 493.4 million with prediabetes [9]. The prevalence rate continues to increase throughout the years specifically for urban India from $8.3 \%$ in 1989 to $18.6 \%$ in 2005 , rural India from $2.2 \%$ in 1989 to $9.2 \%$ in 2005 [10], urban Indonesia from $1.6 \%$ in 1981 to $5.75 \%$ in 1995 , Malaysia from $2.1 \%$ in 1982 to $11 \%$ in 2006 [8], Singapore from $8.2 \%$ in 2004 to $11.3 \%$ in 2010 [11], South Korea from $5.92 \%$ in 2003 to $7.08 \%$ in 2010 [12], and Taiwan from $5.79 \%$ in 2000 to $8.3 \%$ in 2007 [13]. Diabetes is associated with excess mortality [14]. The mortality rate due to diabetes in Singapore increased from $1 \%$ in 2010 to $1.7 \%$ in 2011 [15], and South Korea from $0.017 \%$ in 1995 to $0.025 \%$ in 2005 [16]. The continued increase in the prevalence and mortality associated with type 2 diabetes indicate an unmet medical need. Currently, diabetes accounts for $9 \%$

*Corresponding author: Elvira Zenaida Lansang, Manager, Regional Feasibility and Site Identification, Asia, Quintiles East Asia Pte Ltd, 79 Science Park Drive \#06-08, Cintech IV, Science Park One, Singapore 118264, Tel: (65) 66021634 Fax: (65) 6872 0430; E-mail: elvirazenaida.lansang@quintiles.com

Received November 01, 2013; Accepted January 23, 2014; Published January 28, 2014

Citation: Lansang EZ, Tang V, Ranade S, Caveney E, Ng N, et al. (2014) The Patient Care Pathway for Type 2 Diabetes Mellitus in Asian Countries: Implications on Target Physician Group for Diabetes Clinical Trials. J Diabetes Metab 5: 331 doi:10.4172/2155-6156.1000331

Copyright: (c) 2014 Lansang EZ, et al. This is an open-access article distributed under the terms of the Creative Commons Attribution License, which permits unrestricted use, distribution, and reproduction in any medium, provided the original author and source are credited. 
Citation: Lansang EZ, Tang V, Ranade S, Caveney E, Ng N, et al. (2014) The Patient Care Pathway for Type 2 Diabetes Mellitus in Asian Countries: Implications on Target Physician Group for Diabetes Clinical Trials. J Diabetes Metab 5: 331. doi:10.4172/2155-6156.1000331

of all clinical trials that are ongoing globally [17]. In recent years, Asia has been increasingly engaged in clinical trials to support accelerated recruitment and shorten clinical development timelines [18]. As a result, we see an increase in clinical trials in diabetes globally from $11.4 \%$ in 2007 to $26.5 \%$ in 2009 . Out of 2,484 clinical trials conducted from 2007 to 2010, 28.6\% were conducted within Asia [19].

Patient recruitment for clinical trials becomes more challenging if conducted in areas with a high density of ongoing trials [20]. With the continued increase in diabetes clinical trials in Asia, it is critical to understand the patient care pathway. This will pave the way for the correct identification of institutions and investigators for clinical trials, optimizing recruitment and study timelines. To our knowledge, there is a lack of data on the patient care pathway in Asia. A search of PubMed and through a web search engine i.e. Google using different combinations of the search terms "diabetes", "diabetics", "referral", "care", "pathway", and "Asia" did not reveal any relevant journals. Against this backdrop, the authors' company initiated a survey to better understand the diabetes patient care pathway in Asia.

This paper provides the results of the diabetes patient care pathway survey and provides suggestions on the implications of site selection for diabetes clinical trials.

\section{Methods}

A survey was designed to investigate the patient care pathway for Type 2 Diabetes Mellitus by the Asia Quintiles' feasibility team and Quintiles' physicians. Both groups have a special interest and expertise in the exploratory clinical development of new chemical entities and biosimilar molecules in diabetes. The survey questions were a combination of closed and open-ended questions geared towards determining the main specialty involved in the diagnosis and treatment of diabetes and its associated complications. The survey included questions on the:

- Initial diagnosis of Type 2 Diabetes mellitus

- $\quad$ Specialties treating Type 2 diabetes

- Referral pattern for diabetic micro- and macro-vascular complications

Between March to August 2013, an English-language survey was sent to sites located in 11 Asian countries - China, India, Indonesia, Malaysia, Philippines, Singapore, South Korea, Sri Lanka, Taiwan, Thailand and Vietnam. A site is defined as a healthcare institution that is able to provide care for type 2 diabetic patients. These countries were targeted due to the lack of published information on the diabetic patient care pathway in Asia.

Convenience sampling was performed to identify sites for inclusion in the survey from Quintiles internal database. Each site chosen had the capability to diagnose/treat diabetes patients or had previous experience with Quintiles in conducting diabetes trials. Both generalist and specialist sites were contacted in each country. At each site, the survey was completed by physicians who have either participated in Quintiles' diabetes clinical trials, have a large pool of diabetes patients or who were recommended by the clinical research center. Respondents were requested to consider the patient care pathway for their institution. The survey forms were sent by email or posted directly to physicians at the sites. Appropriate follow ups were made to encourage return of completed surveys.
Answers were based on each physician's recall of how patients in their practice were initially diagnosed with Type 2 diabetes, which specialties were involved in the management, and the referral pattern for diabetes complications. In September 2013, the responses from the completed survey forms were entered into Click Tools, a web-based central repository. A descriptive data analysis was performed.

\section{Results}

Eighty-seven sites were sent surveys across 11 Asian countries. Fifty-six percent (49/87) returned the surveys (Table 1).

Majority of the physicians who participated in the survey identified themselves as endocrinologists or diabetologists (Table 2).

Physicians were asked to indicate the top 3 medical specialties at their site that diagnose type 2 diabetes mellitus for the first time. Figure 1 illustrates that internal medicine; general practice and endocrinology are the most common specialties providing the initial diagnosis of type 2 diabetes mellitus in Asian countries.

Various treatment options, both pharmacologic and nonpharmacologic, are available for patients diagnosed with diabetes. Patients can be treated with medical nutrition therapy and physical activity, various oral anti-hyperglycemic agents, insulin and/or GLP-1 agonists [21,22]. The survey focused mainly on the medical specialties that are involved in the different treatment regimens used for diabetic patients in Asia. Endocrinologists, internists and general practitioners manage majority of diabetic patients whose glucose levels are sufficiently controlled through lifestyle interventions and those needing oral anti-hyperglycemic agents. Endocrinologists and

\begin{tabular}{|l|c|c|c|}
\hline Country & $\begin{array}{c}\text { Number of Sites } \\
\text { Contacted }\end{array}$ & $\begin{array}{c}\text { Number of } \\
\text { Responses }\end{array}$ & $\begin{array}{c}\text { Response } \\
\text { Rate }\end{array}$ \\
\hline China & 9 & 9 & $100 \%$ \\
\hline India & 6 & 4 & $67 \%$ \\
\hline Indonesia & 16 & 4 & $25 \%$ \\
\hline Malaysia & 8 & 6 & $75 \%$ \\
\hline Philippines & 8 & 5 & $63 \%$ \\
\hline Singapore & 7 & 3 & $43 \%$ \\
\hline South Korea & 4 & 4 & $100 \%$ \\
\hline Sri Lanka & 2 & 1 & $50 \%$ \\
\hline Taiwan & 12 & 4 & $33 \%$ \\
\hline Thailand & 9 & 5 & $56 \%$ \\
\hline Vietnam & 6 & 4 & $67 \%$ \\
\hline Total & 87 & 49 & $56 \%$ \\
\hline
\end{tabular}

Table 1: Response rate to the survey.

\begin{tabular}{|l|c|c|}
\hline Specialty & N & Percentage \\
\hline Endocrinologist & 28 & $57 \%$ \\
\hline Diabetologist & 12 & $24 \%$ \\
\hline General Practitioner (GP) & 7 & $14 \%$ \\
\hline Internist (Internal Medicine) $)^{\star \star}$ & 7 & $14 \%$ \\
\hline Family Practice & 4 & $8 \%$ \\
\hline Cardiologist & 3 & $6 \%$ \\
\hline Neurologist & 1 & $2 \%$ \\
\hline
\end{tabular}

*A physician may have more than one medical specialty.

${ }^{* *} \mathrm{~A}$ GP is a medical practitioner who treats acute and chronic illnesses and provides preventive care and health education for all ages and all sexes. They have particular skills in treating people with multiple health issues and comorbidities. An internist is a physician trained in internal medicine [21]

Table 2: Specialties of the 49 physicians who responded to the survey*. 
Citation: Lansang EZ, Tang V, Ranade S, Caveney E, Ng N, et al. (2014) The Patient Care Pathway for Type 2 Diabetes Mellitus in Asian Countries: Implications on Target Physician Group for Diabetes Clinical Trials. J Diabetes Metab 5: 331. doi:10.4172/2155-6156.1000331

diabetologists manage majority of patients requiring insulin and GLP1 agonists. Next to endocrinologists/diabetologists, the internists also see diabetics needing insulin to control their glucose levels (Table 3).

All respondents reported having diabetic patients with complications. Figure 2 shows the medical specialties providing the initial diagnosis of a diabetic complication.

Figure 3 illustrates the different specialties to which patients who are diagnosed with diabetic complications are referred to for further management.

The survey also explored the conditions warranting referral of a diabetic complication to another specialist. Verbatim responses provided by physicians were grouped into similar categories. Table 4 summarizes the findings.

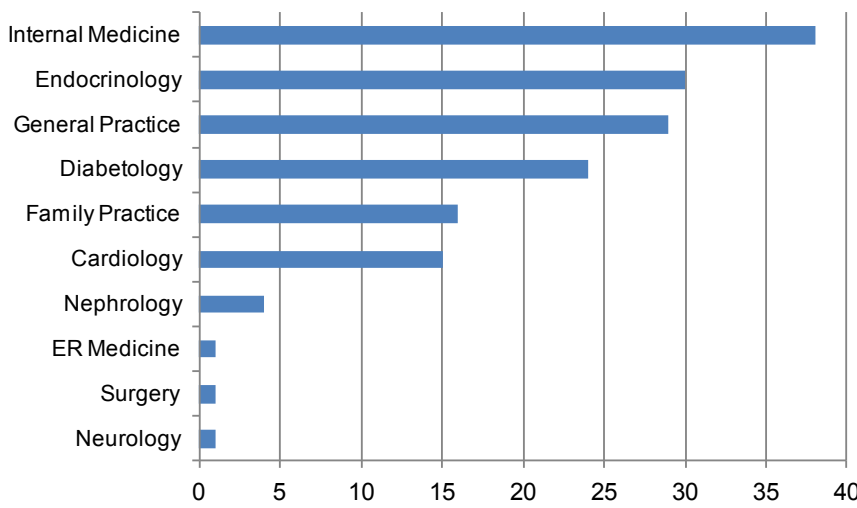

Figure 1: Top 3 medical specialties that make the initial diagnosis of type 2 diabetes mellitus at each site $(\mathrm{N}=49)$.

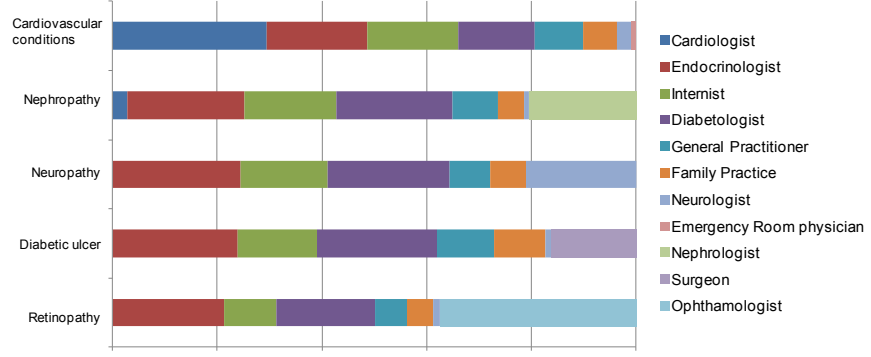

Figure 2: Medical specialties involved in the diagnosis of each diabetic complication at each institution $(\mathrm{N}=49)$.

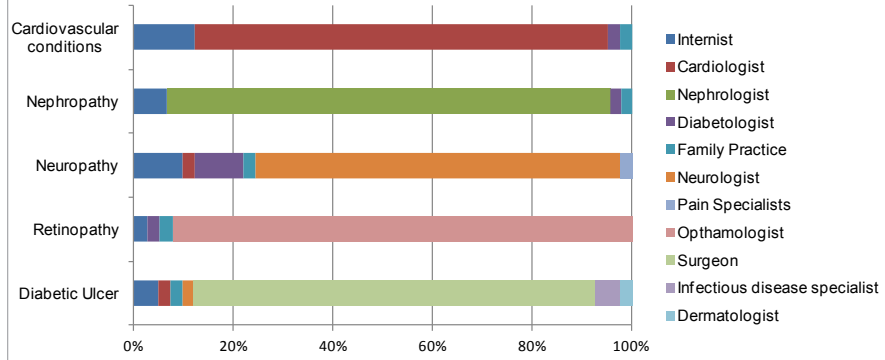

Figure 3: Medical specialties to whom patients with diabetic complications are referred to at each institution $(\mathrm{N}=49)$.

\section{Discussion}

Type 2 diabetes is a chronic disease and people with diabetes should ideally receive medical care from a physician-coordinated team [22]. Several clinical practice guidelines for the management of diabetes and its associated complications are available. These guide treatment decisions for diabetic patients. However, these guidelines do not always dictate the medical specialty of the health care provider involved in the care of the patient.

An analysis of the global pharmaceutical market shows that diabetes will have the second highest level of spending on medicines by 2016, next to cancer [23]. Furthermore, diabetes ranks second among the global priority diseases in terms of pharmaceutical pipeline products under development [24]. Due to these trends, it is anticipated that the number of diabetic clinical trials in Asia will continue to increase.

Conducting site-level feasibility is one of the important stages when planning for a clinical trial. One of the most important components of feasibility is to assess a site's recruitment potential, specifically the anticipated subjects that can be recruited into the trial [25-27]. Knowing the patient care pathway for diabetes in Asia will help in determining the sites and investigators that can be approached when conducting feasibility for a particular clinical trial. A search of published literature revealed no data describing the diabetes patient care pathway in Asia.

Type 2 diabetes clinical trials not only seek patients with differing backgrounds of diabetes treatment, but increasingly, studies search for patients who have developed acute or long term complications. For example, eligibility criteria may range from those who have not received prior pharmacologic treatment; have glucose levels that are not adequately controlled by metformin or other oral anti-hyperglycemic agents; are receiving insulin; or have various micro- or macro-vascular complications such as previous coronary artery disease, nephropathy, neuropathy, or retinopathy. The survey showed that internists, general practitioners, and endocrinologists provide the initial diagnosis of type 2 diabetes. Consequently, these are also the medical specialties that see majority of patients who are not on any pharmacologic therapy. Within Asia, there are inter-country variations in the medical specialty providing the initial diagnosis of diabetes. The survey revealed that endocrinologists/diabetologists are involved in the initial diagnosis of many diabetic patients in China, India and South Korea. In Indonesia, Malaysia and Thailand, the initial diagnosis is usually made by general practitioners/family medicine specialists. When selecting sites for clinical trials requiring treatment naive diabetic patients, it is important to consider general practitioners and internists as potential investigators. Unfortunately this brings a certain amount of risk as one of the main challenges with conducting clinical trials in developing countries is the lack of experienced investigators and the limited research infrastructure [28]. Majority of primary healthcare sites in Asia do not have the sufficient infrastructure for clinical trials. For such countries, the possibility of being referral centers needs to be explored. One of the characteristics of a high performing site located in India for a global diabetes clinical trial utilized networks to create informal links with general practitioners, resulting in a very positive effect [18].

Table 3 shows that diabetologists/endocrinologists manage majority of patients in Asia who receive pharmacologic therapy, including oral anti-hyperglycemic agents $(80 \%)$, insulin $(82 \%)$ and GLP-1 agonists (70\%). While the disparity between endocrinologists and GPs/ internists is less among diabetic patients on oral antihyperglycemic agents, it is clear that majority of patients on insulin 
Citation: Lansang EZ, Tang V, Ranade S, Caveney E, Ng N, et al. (2014) The Patient Care Pathway for Type 2 Diabetes Mellitus in Asian Countries: Implications on Target Physician Group for Diabetes Clinical Trials. J Diabetes Metab 5: 331. doi:10.4172/2155-6156.1000331

Page 4 of 6

\begin{tabular}{|c|c|c|c|c|}
\hline & Lifestyle Intervention & Oral Anti-hyperglycemic Agents & Insulin & GLP-1 Agonists \\
\hline General Practitioner & $61 \%$ & $65 \%$ & $31 \%$ & $8 \%$ \\
\hline Internist & $65 \%$ & $76 \%$ & $51 \%$ & $22 \%$ \\
\hline Endocrinologist/ Diabetologist & $78 \%$ & $82 \%$ & $84 \%$ & $73 \%$ \\
\hline Cardiologist & $51 \%$ & $59 \%$ & $39 \%$ & $18 \%$ \\
\hline Nephrologist & $35 \%$ & $45 \%$ & $35 \%$ & $8 \%$ \\
\hline Surgeon & $22 \%$ & $27 \%$ & $16 \%$ & $4 \%$ \\
\hline Neurologist & $4 \%$ & $2 \%$ & $2 \%$ & - \\
\hline
\end{tabular}

*Each medical specialty may prescribe multiple therapies for a patient

Table 3: Medical specialties managing diabetes according to administered treatment $(\mathrm{N}=49)^{*}$.

or GLP-1 agonists are managed by endocrinologists/diabetologists in Asia. Furthermore, general practitioners manage diabetics through lifestyle interventions or oral anti-hyperglycemic agents. On the other hand, specialists such as endocrinologists/diabetologists equally use oral anti-hyperglycemic agents and insulin, in addition to lifestyle interventions. This is similar to the findings of Britt et al. who evaluated 261 type 2 diabetics in general practice in Australia. Almost two-thirds of all diabetics had their insulin therapy initiated by a specialist and for just under $20 \%$, it was initiated by a GP alone [29]. According to the American Diabetes Association 2013 Position Statement, metformin (if not contraindicated) is the preferred initial pharmacologic agent for newly diagnosed type 2 diabetics. Insulin may be considered for newly diagnosed patients whose glucose levels are markedly elevated or who are highly symptomatic. Furthermore, insulin or GLP-1 agonists may be added for patients who still have poor glucose control despite 3 to 6 months of treatment with maximum tolerated doses of non-insulin therapy [22]. These recommendations support the referral of patients who have poor glucose control or who develop complications from their primary care physicians to other specialties.

Diabetic complications are either macrovascular or microvascular in nature. According to Geiss et al., more than $75 \%$ of all mortality among diabetic persons is related to cardiovascular disease [30]. One half of patients with type 2 diabetes die prematurely of a cardiovascular cause and approximately $10 \%$ die of renal failure [31]. Furthermore, studies on Western populations reveal that cardiovascular [32] and kidney complications [33] are most prevalent. In China, the most common complication is also cardiovascular disease, with a prevalence of $30.1 \%$ [34]. Although not mentioned specifically by any of the respondents, one emerging complication among diabetic patients is the increased risk of bone fractures. Asians with diabetes, like Westerners, experience an increased risk of hip fracture [35]. A retrospective study by Yaturu et al. suggests that thiazolidinedione treatment may be a risk factor for the development of fractures in diabetics [36]. It is recommended that this be investigated further in subsequent studies.

Figures 2 and 3 illustrate the percentage distribution of the different medical specialties that diagnose diabetic complications and the specialties to which patients are referred to. Cardiovascular and ophthalmologic conditions are diagnosed by cardiologists and ophthalmologists respectively. Not surprisingly, they are also the specialists who manage these complications. The distinction among specialties that diagnose nephropathy, peripheral neuropathy and foot ulcers is less compared to cardiovascular and ophthalmologic conditions. Diabetologists/endocrinologists diagnose majority of patients with nephropathy, peripheral neuropathy and diabetic ulcers, with more targeted specialists such as nephrology, neurologists and surgeons coming in second. While different specialties are able to diagnose a complication, Figure 3 clearly illustrates that there is a main specialty where majority of patients with a particular complication are referred to.
Table 4 details the different conditions warranting referral to a different specialist. The inclusion of GPs, internists, family practice doctors, endocrinologists and diabetologists as principal investigator in a clinical trial should be considered for protocols needing patients with less severe complications. Targeted specialists such as cardiologists, nephrologists, surgeons and ophthalmologists are recommended for the more severe cases. In particular, studies with cardiovascular or nephropathy endpoints are common in the development of antidiabetic compounds. As such, cardiologists and nephrologists may in some countries be the most important investigators for these types of studies. Majority of patients across Asia, with painful diabetic neuropathy are primarily managed by diabetologists/endocrinologists, and referred to specialist/ neurologist depending on the severity of the complication. The survey also revealed that in South Korea, patients whose symptoms progress, continue to be treated by the diabetologists/ endocrinologists. The involvement of different specialties in the management of the diabetic patient highlights the complexity of this disease and the need for specialist care. The Diabetes UK Task and Finish Group Report recommends that specialist teams provide direct care for diabetics with complex needs that cannot be met within the skill competencies of the general practice team [37]. This helps pave the way for a high quality integrated diabetes service [38].

Some developed countries such as New Zealand, Ireland and the United Kingdom utilize care pathways that guide decisions and timing for the diagnosis, intervention and referral of diabetes patients. The implementation of care pathways have been shown to reduce variability in clinical practice, reduce healthcare costs and improve patient outcomes [39]. In Ireland and the United Kingdom, the use of care pathways hopes to reduce patient waiting times and increase patient access to services [37,40]. Despite the lack of similar published literature specific to Asia, the survey results are evidence of the multidisciplinary approach to the management of diabetes in Asia. Studies have described the benefits of using this approach towards achieving improved metabolic control [41-43]. When selecting sites for diabetes clinical trials, it is important to understand the subset of diabetic patients required and consequently assess the medical specialties that would need to be targeted.

\section{Limitation}

Consistent with voluntary surveys, the authors were only able to analyze responses from those who were interested in replying. Therefore, we cannot exclude that there may be additional opinions and points of view from those that did not respond. The authors have gathered responses from 11 Asian countries. However, given the relatively limited number of responses received, the findings may be viewed as suggestions, rather than firm conclusions. 
Citation: Lansang EZ, Tang V, Ranade S, Caveney E, Ng N, et al. (2014) The Patient Care Pathway for Type 2 Diabetes Mellitus in Asian Countries: Implications on Target Physician Group for Diabetes Clinical Trials. J Diabetes Metab 5: 331. doi:10.4172/2155-6156.1000331

Page 5 of 6

\begin{tabular}{|c|c|c|c|}
\hline Complication & Conditions of Referral & $\mathbf{N}^{*}$ & $\%$ ** \\
\hline $\begin{array}{l}\text { Cardiovascular } \\
\text { Conditions }\end{array}$ & 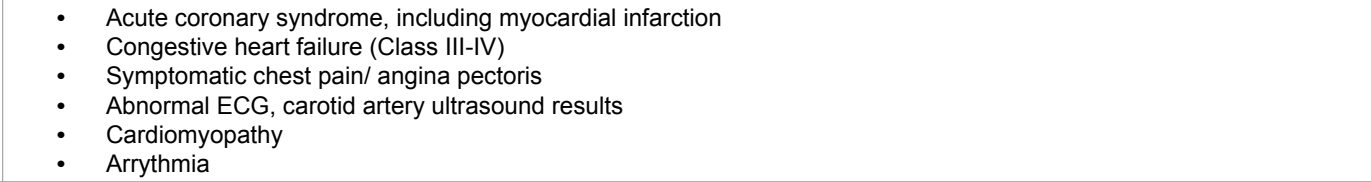 & $\begin{array}{l}20 \\
6 \\
6 \\
3 \\
2 \\
2\end{array}$ & $\begin{array}{l}51.3 \\
15.4 \\
15.4 \\
7.7 \\
5.1 \\
5.1\end{array}$ \\
\hline Nephropathy & $\begin{array}{l}\text { - Azotemia such as those with increased creatinine ( }>150-300 \mu \mathrm{mol} / \mathrm{L}) \text {, or those with } \mathrm{GFR}<30-40 \\
\text { - } \quad \text { Uremia, including those approaching end-stage renal disease/ needing dialysis/ with hyperkalemia } \\
\text { Proteinuria }(>1.5-3 \mathrm{~g} / \text { day) }\end{array}$ & $\begin{array}{l}16 \\
16 \\
11\end{array}$ & $\begin{array}{l}37.2 \\
37.2 \\
25.6\end{array}$ \\
\hline Peripheral Neuropathy & $\begin{array}{l}\text { - Limb weakness/numbness/tingling of extremities } \\
\text { - } \quad \text { Uncontrolled neuropathic pain despite conventional treatment } \\
\text { - Exacerbation of pain/Myodynia/Neuralgia }\end{array}$ & $\begin{array}{l}12 \\
4 \\
4\end{array}$ & $\begin{array}{l}60 \\
20 \\
20\end{array}$ \\
\hline $\begin{array}{l}\text { Cerebrovascular } \\
\text { Conditions }\end{array}$ & $\begin{array}{ll}\text { - } & \text { Stroke } \\
\text { - } & \text { Vertigo }\end{array}$ & $\begin{array}{l}3 \\
1\end{array}$ & $\begin{array}{l}75 \\
25\end{array}$ \\
\hline Diabetic Ulcer & $\begin{array}{ll}\text { - } & \text { Non-healing ulcer (size progression) } \\
\text { - } & \text { Onset/ suspicion of infection } \\
\text { - } & \text { Gangrene limb } \\
\text { - } & \text { Osteomyelitis } \\
\text { - } & \text { Cellulitis } \\
\text { - } & \text { Suspicious for ischemia }\end{array}$ & $\begin{array}{l}10 \\
7 \\
7 \\
3 \\
2 \\
1 \\
1\end{array}$ & $\begin{array}{r}32.3 \\
22.6 \\
22.6 \\
9.7 \\
6.4 \\
3.2 \\
3.2\end{array}$ \\
\hline Retinopathy & $\begin{array}{l}\text { - Ocular fundus changes (by fundoscopy or fundus photography) - such as but not limited to: NPDR (Non Proliferative } \\
\text { Diabetic Retinopathy), PPDR (Pre-proliferative Diabetic Retinopathy), PDR (Proliferative Diabetic Retinopathy), } \\
\text { Advanced (Gr III-IV) Diabetic Retinopathy, Macular Edema, Retinal Detachment, Fundus hemorrhage } \\
\text { - } \quad \text { Acute decrease in vision in short duration } \\
\text { - } \quad \text { Routine annual eye check-up } \\
\text { - } \quad \text { Cataract requiring surgical intervention } \\
\text { Suspected glaucoma }\end{array}$ & $\begin{array}{l}8 \\
6 \\
3 \\
2\end{array}$ & $\begin{array}{r}36.7 \\
26.7 \\
20 \\
10 \\
6.7\end{array}$ \\
\hline
\end{tabular}

${ }^{*} \mathrm{~N}$ refers to the number of times physicians have mentioned each condition warranting referral to another specialist

**Percentage refers to the number of times physician cite each condition of referral over all conditions of referrals mentioned

Table 4: Conditions warranting referral of diabetic complications to another specialist.

\section{Conclusion}

In summary, understanding the diabetes patient care pathway has led to some suggestions on the target physician group for clinical trials in Asia, namely (1) internists and primary healthcare physicians may be potential investigators or referral sites for clinical trials requiring treatment naïve diabetic patients; (2) diabetologists/endocrinologists should be considered for clinical trials needing patients on pharmacologic treatment, whether oral or insulin; (3) internists may play a role in trials that involve patients on oral anti-hyperglycemic agents and on insulin; (4) the severity of diabetic complications impacts the medical specialty of the physician treating patients; and (5) cardiologists and nephrologists are important considerations for studies with cardiovascular or nephrology related outcomes.

\section{Acknowledgements}

The authors would like to thank Quintiles team members across the region that helped reach out to sites in order to complete the survey, particularly Audrey Ho Chris Zhang, EunJung Lee, Henu Tonang, HyunSuk Hong, Liling Chen, Manish Thadhani, Maria Rolyn Viaje, NaRae Baek, Piyanuch Tiativiriyakul, Siew Lee Goh, Suqiong Wang, Yi Chin Ong, Xu Su, Adeline Sng, Jessica Lee and Josey Lee.

\section{Disclosure}

$\checkmark$ Tang is a former contract employee of Quintiles. She has helped gather data and literature for this research. Ms Tang has not received any additional payment from Quintiles for the conduct of this research. The other authors have no conflicts of interest in this work.

\section{References}

1. Rotter T, Kinsman L, James El, Machotta A, Gothe H, et al. (2010) Clinical pathways: effects on professional practice, patient outcomes, length of stay and hospital costs. Cochrane Database of Systematic Reviews 3.

2. Peters JL, Anderson R, Hyde C (2013) Development of an economic evaluation of diagnostic strategies: the case of monogenic diabetes. BMJ Open 3.

3. Goenka N, Vora J (2011) Commissioning specialist diabetes care. Practical Diabetes International 28: 58-59.
4. Bi Y, Wang T, Xu M, Xu Y, Li M, et al. (2012) Advanced research on risk factors of type 2 diabetes. Diabetes Metab Res Rev 28: 32-39.

5. Ramachandran A, Ma RC, Snehalatha C (2010) Diabetes in Asia. Lancet 375 408-418.

6. Chan JC, Malik V, Jia W, Kadowaki T, Yajnik CS, et al. (2009) Diabetes in Asia: epidemiology, risk factors, and pathophysiology. JAMA 301: 2129-2140.

7. OECD/ World Health Organization (2012) Health at a glance: Asian/Pacific: 34

8. Ramachandran A, Snehalatha C, Shetty AS, Nanditha A (2012) Trends in prevalence of diabetes in Asian countries. World J Diabetes 3: 110-117.

9. Xu Y, Wang L, He J, Bi Y, Li M, et al. (2013) Prevalence and control of diabetes in Chinese adults. JAMA 310: 948-959.

10. Ramachandran A, Mary S, Yamuna A, Murugesan N, Snehalatha C (2008) High prevalence of diabetes and cardiovascular risk factors associated with urbanization in India. Diabetes Care 31: 893-898.

11. National Registry of Diseases Office (2011) Information paper on diabetes in Singapore. Health Promotion Board: 1-6.

12. Task Force Team for Basic Statistical Study of Korean Diabetes Mellitus of Korean Diabetes Association, Park IB, Kim J, Kim DJ, Chung CH, et al. (2013) Diabetes epidemics in Korea: Reappraise, nationwide survey for diabetes "diabetes in Korea 2007". Diabetes and Metabolism Journal 37: 233-239.

13. Lin CC $\mathrm{Li} \mathrm{Cl}$, Hsiao CY, Liu CS, Yang SY, et al. (2013) Time trend analysis of the prevalence and incidence of diagnosed type 2 diabetes among adults in Taiwan from 2000 to 2007: a population-based study. BMC Public Health 13: 318

14. Roper NA, Bilous RW, Kelly WF, Unwin NC, Connolly VM (2001) Excess mortality in a population with diabetes and the impact of material deprivation: longitudinal, population based study. BMJ 9: 1389-1393.

15. Ministry of Health (2012) Principal causes of death

16. (2006) 2005 Statistical results about cause of death. National Statistical Office.

17. ClinicalTrials.gov protocol registration system. PRS information.

18. Abraham A, Jones J, Vikram S (2010) How to minimize low enrolling sites: a case study in diabetes. Perspect Clin Res 1: 25-28. 
Citation: Lansang EZ, Tang V, Ranade S, Caveney E, Ng N, et al. (2014) The Patient Care Pathway for Type 2 Diabetes Mellitus in Asian Countries: Implications on Target Physician Group for Diabetes Clinical Trials. J Diabetes Metab 5: 331. doi:10.4172/2155-6156.1000331

Page 6 of 6

19. Lakey WC, Barnard K, Batch BC, Chiswell K, Tasneem A, et al. (2013) Are current clinical trials in diabetes addressing important issues in diabetes care? Diabetologia 56: 1226-1235.

20. Wai K, Saldanha LM, Lansang EZ, Nayak S, Sule A, et al. (2013) Case series of feasibility considerations that impact operational delivery strategy in the highly competitive rheumatoid arthritis space in Asia. Open Access Journal of Clinical Trials 5: 33-38.

21. Malaysian General Practitioner.

22. American Diabetes Association (2013) Standards of medical care in diabetes--2013. Diabetes Care 36: S11-66.

23. (2012) The global use of medicines: outlook through 2016. IMS Institute for Healthcare Informatics: 7

24. (2012) The global use of medicines: outlook through 2016. IMS Institute for Healthcare Informatics: 16.

25. Rajadhyaksha V (2010) Conducting feasibilities in clinical trials: an investment to ensure a good study. Perspect Clin Res 1: 106-109.

26. Harper BD, Zuckerman D (2006) Critical success factors for planning for site selection and patient recruitment planning. BioExecutive International 2: S16-S28.

27. Warden D, Trivedi MH, Greer TL, Nunes E, Grannemann BD, et al. (2012) Rationale and methods for site selection for a trial using a novel intervention to treat stimulant abuse. Contemp Clin Trials 33: 29-37.

28. Devasenapathy N, Singh K, Prabhakaran D (2009) Conduct of clinical trials in developing countries: a perspective. Curr Opin Cardiol 24: 295-300.

29. Britt H, Miller GC, Charles J, Henderson J, Bayram C, et al. (2009) General practice activity in Australia, 2008-09. General practice series no. 25. Cat. no. GEP 25. Canberra: AlHW.

30. Geiss LS, Herman WH, Smith PJ (1995) Mortality in non-insulin dependent diabetes. National Institute of Health: 233-255.

31. van Dieren S, Beulens JW, van der Schouw YT, Grobbee DE, Neal B (2010) The global burden of diabetes and its complications: an emerging pandemic. Eur J Cardiovasc Prev Rehabil 17: S3-8.
32. Morgan CL, Peters JR, Currie CJ (2010) The changing prevalence of diagnosed diabetes and its associated vascular complications in a large region of the UK. Diabet Med 27: 673-678.

33. Dannenbaum D, Kuznina E, Kejeune P, Torrie J, Gangbe M (2008) Prevalence of diabetes and diabetes-related complications in first nations communities in northern Quebec (Eeyou Istchee), Canada. Canadian Journal of Diabetes 32: 46-52.

34. Liu Z, Fu C, Wang W, Xu B (2010) Prevalence of chronic complications of type 2 diabetes mellitus in outpatients - a cross-sectional hospital based survey in urban China. Health Qual Life Outcomes 8: 62.

35. Koh WP, Wang R, Ang LW, Heng D, Yuan JM, et al. (2010) Diabetes and risk of hip fracture in the Singapore Chinese Health Study. Diabetes Care 33 1766-1770.

36. Yaturu S, Bryant B, Jain SK (2007) Thiazolidinedione treatment decreases bone mineral density in type 2 diabetic men. Diabetes Care 30: 1574-1576.

37. Goenka N, Turner B, Vora J; Diabetes UK Task and Finish group (2011) Commissioning specialist diabetes services for adults with diabetes: summary of a Diabetes UK Task and Finish group report. Diabet Med 28: 1494-1500.

38. (2007) Integrated care in the reforming NHS â€“ ensuring access to high-quality care for all people with diabetes. A Joint Position Statement of Diabetes UK.

39. (2012) Care pathways for long-term conditions: using type 2 diabetes as an example. Best Practice Journal 47: 38-41.

40. (2011) Report and recommendations on patient referrals from general practice to outpatient and radiology services, including the national standard for patient referral information. Health Information and Quality Authority.

41. Maislos M, Weisman D (2004) Multidisciplinary approach to patients with poorly controlled type 2 diabetes mellitus: a prospective, randomized study. Acta Diabetol 41: 44-48.

42. Bayless M, Martin C (1998) The team approach to intensive diabetes management. Diabetes Spectrum 11: 33-37.

43. (1995) Implementation of treatment protocols in the Diabetes Control and Complications Trial. Diabetes Care 18: 361-376. 